\title{
Fungos associados às sementes de ipê-amarelo (Tabebuia serratifolia ) e ipê-roxo (Tabebuia impetiginosa): incidência, efeito na germinação e transmissão para as plântulas
}

\author{
Luana da Silva Botelho ${ }^{1}$, Maria Heloisa Duarte Moraes ${ }^{1}$, José Otávio Machado Menten ${ }^{1}$
}

${ }^{1}$ ESALQ/USP, C.P. 09, Piracicaba, SP, CEP 13418-900, e-mail: luanasbotelho@ yahoo.com.br.

Autor para correspondência: Luana da Silva Botelho

Data de chegada: 30/11/2006. Aceito para publicação em: 29/05/2007.

\section{RESUMO}

Botelho, L.S.; Moraes, M.H.D., Menten, J.O.M. Fungos associados às sementes de ipê-amarelo (Tabebuia serratifolia) e ipê-roxo (Tabebuia impetiginosa): incidência, efeitos na germinação e transmissão para as plântulas. Summa Phytopathologica, v.34, n.4, p.343-348, 2008

Este trabalho teve como objetivos fazer um levantamento dos fungos presentes em oito amostras de sementes de ipê-amarelo (Tabebuia serratifolia) e ipê-roxo (T. impetiginosa) coletadas nas regiões de Piracicaba, Mogi-Guaçu e sul de Minas Gerais (Lavras, Ijaci e Itumirim) e determinar os possíveis prejuízos na produção de mudas dessas espécies. O método utilizado para o teste de sanidade foi o de papel de filtro e, para o de germinação, utilizou-se caixa tipo gerbox com substrato de papel à temperatura de $30^{\circ} \mathrm{C}$ sob regime de luz constante. As sementes, tanto no teste de sanidade quanto no de germinação, foram subdivididas sendo uma parte submetidas à assepsia superficial com hipoclorito de sódio e a outra não. Avaliou-se a transmissão dos fungos através de lesões encontradas nas plântulas, durante o teste de germinação. Foram identificados e quantificados dezesseis fungos: Cladosporium sp., Alternaria alternata, Epicoccum sp., Phoma sp., Geotrichum sp., Penicillium sp., Trichothecium sp., Phomopsis sp., Drechslera sp., Aspergillus spp., Curvularia sp., Fusarium spp., Macrophomina phaseolina, Nigrospora sp., Lasiodiplodia theobromae e Septoria sp. De maneira geral, a assepsia proporcionou redução drástica na incidência de todos os fungos, em ambas espécies, com uma taxa média de $90 \%$, podendo-se inferir que a maioria dos fungos estava contaminando as sementes. Os fungos não interferiram diretamente na porcentagem de plântulas normais e a assepsia reduziu a germinação em $64 \%$, demonstrando ser fitotóxica. $\mathrm{Na}$ transmissão observou-se, em média, $17 \%$ e $10 \%$ de plântulas com sintomas, nas amostras sem assepsia e com assepsia, respectivamente. Os fungos mais freqüentes transmitidos pelas sementes de ipê-amarelo e roxo foram: Alternaria alternata, Fusarium spp., Aspergillus spp., Phoma sp. e Phomopsis sp.

Palavras-chave adicionais: plântulas infectadas, sementes florestais, assepsia.

\begin{abstract}
Botelho, L.S.; Moraes, M.H.D., Menten, J.O.M.. Fungi associated to the seeds of ipê-amarelo (Tabebuia serratifolia) and ipê-roxo (Tabebuia impetiginosa): incidence, germination effect and seedlings transmission. Summa Phytopathologica, v.34, n.4, p.343-348, 2008

The aim of this research was to check out what kind of fungi was present in eight samples of ipê-amarelo seeds (Tabebuia serratifola) and ipê-roxo ( $T$. impetiginosa) collected from the region of Piracicaba, Mogi-Guaçu and south of Minas Gerais (Lavras, Ijaci e Itumirim) and to determine the possible damages in the seedlings production of those species. The method used for the health test was the blotter test and, for the germination, gerbox had been used with paper substratum at temperature of $30^{\circ} \mathrm{C}$ under constant light regime. The seeds, as much in the health test as in the germination, tests were sub sampled in one part that was submitted to superficial desinfestation with sodium hypochlorite of, and one part that wasn't. The fungi transmission had been evaluated through lesions found in the seedlings, during the germination test. Sixteen fungi had been identified and quantified: Cladosporium sp., Alternaria alternata, Epicoccum sp.,

Phoma sp., Geotrichum sp., Penicillium sp., Trichothecium sp., Phomopsis sp., Drechslera sp., Aspergillus spp., Curvularia sp., Fusarium spp., Macrophomina phaseolina, Nigrospora sp., Lasiodiplodia theobroma and Septoria sp. In general, the asepsis provided a drastic reduction in the incidence of all fungi, in both species, with an avarege rate of $90 \%$. Like this, it can be inferred that most of the fungi was contaminating the seeds. The percentage of normal seedlings had not been interfered directly by the fungi. The asepsis reduced the germination in $64 \%$, indicatinwas fitotoxic. In the transmission it had been observed, on average, $17 \%$ and $10 \%$ of seedlings with symptoms, in the samples without asepsis and with asepsis, respectively. The most frequent fungi transmitted by the ipê-amarelo and ipê-roxo seeds had been: Alternaria alternata, Fusarium spp., Aspergillus spp., Phoma sp. and Phomopsis sp.
\end{abstract}

Keywords: Infected seedlings, Forest seeds, Asepsis

A importância dos patógenos associados às sementes é evidente; porém, são escassas as informações a respeito da qualidade sanitária das sementes de espécies florestais nativas, utilizadas atualmente.

Os gêneros de fungos que têm sido encontrados em sementes de várias florestais, no Brasil, são Alternaria, Aspergillus, Lasiodiplodia Chaetomium, Cladosporium, Curvularia, Cylindrocladium, Diplodia, Epicoccum, Fusarium, Drechslera, Macrophomina, Monocillium, Nigrospora, Oidiodendron, Penicillium, Pestalotiopsis, Phoma, 
Pithomyces, Peyronellacea, Rhizoctonia e Trichoderma (5, 13, 20).

Devido à importância dada ao gênero Tabebuia, a qualidade sanitária das sementes de suas espécies têm sido estudada. Vários fungos, relatados em associação com outras diferentes espécies florestais nativas, foram detectados nas sementes deste gênero. Carvalho e Muchovej (6), trabalhando com sementes de ipê-amarelo (Tabebuia serratifolia) e outras espécies, constataram como os principais gêneros de fungos Penicillium, Aspergillus e Fusarium. Em sementes de ipê-amarelo (T. serratifolia), os principais gêneros de fungos detectados foram Alternaria, Rhizopus, Curvularia, Cladosporium e Fusarium sp. (27).

Esses trabalhos relatam, na maioria das vezes, apenas os fungos associados a essas sementes, sendo necessário um estudo complementar, aliado a outras áreas de tecnologia e produção de sementes, principalmente visando os efeitos que os fungos podem causar às sementes e mudas.

$\mathrm{O}$ efeito de fungos mais freqüentemente detectados em sementes de ipê-roxo e ipê-amarelo foi verificado por Sales (26). Constatou-se que Alternaria alternata e Phomopsis sp. reduziram a germinação e o desenvolvimento das plântulas de ipê-amarelo; para o ipê-roxo não foi detectado efeito dos fungos nas sementes. Em sementes de ipêrosa (Tabebuia pentaphylla), Fusarium sp. e Phoma sp., foram os principais fungos responsáveis pelo maior número de sementes mortas (25).

Os objetivos do trabalho foram identificar e quantificar fungos transportados em amostras de sementes de ipê-amarelo (T. serratifolia) e ipê-roxo (T. impetiginosa), provenientes de diferentes localidades e períodos de coleta distintos, e avaliar o efeito desses fungos na germinação e transmissão para as plântulas.

\section{MATERIAL E MÉTODOS}

O presente trabalho foi desenvolvido no laboratório de Patologia de Sementes do Depto. de Entomologia, Fitopatologia e Zoologia Agrícola da Escola Superior de Agricultura "Luiz de Queiroz"/USP. Utilizaram-se três amostras de sementes de ipê-amarelo (Tabebuia serratifolia) e cinco de ipê-roxo (T. impetiginosa), provenientes de localidades e épocas de coleta distintas (Tabela 1$)$. As sementes foram armazenadas em sacos plásticos na câmara fria e seca $\left(20^{\circ} \mathrm{C}\right.$ e $40 \%$ U.R.).

Foi determinado o teor de água das sementes no laboratório de Tecnologia de Sementes, utilizando-se o método de estufa a $105 \pm$ $3^{\circ} \mathrm{C}$, por 24 horas (3). Utilizaram-se duas repetições de $1 \mathrm{~g}$ de sementes por amostra e os resultados foram expressos em porcentagem.

A qualidade sanitária das sementes foi analisada utilizando-se o método do papel de filtro, sendo que uma subamostra foi submetida à

Tabela 1. Caracterização das amostras de sementes ipê-amarelo (Tabebuia serratifolia) e ipê-roxo (T. impetiginosa) provenientes de localidades e épocas de coleta distintas

\begin{tabular}{lllc}
\hline Amostra & Espécie & Localidade & Época de Coleta \\
\hline AmrJ2 & T. serratifolia & Ijaci-MG & 2002 \\
AmrI2 & T. serratifolia & Itumirim-MG & 2002 \\
AmrI3 & T. serratifolia & Itumirim-MG & 2003 \\
RoxL2 & T. impetiginosa & Lavras-MG & 2002 \\
RoxP3 & T. impetiginosa & Piracicaba-SP & 2003 \\
RoxL3 & T. impetiginosa & Lavras-MG & 2003 \\
RoxG5 & T. impetiginosa & Mogi-Guaçu-SP & 2005 \\
RoxL5 & T. impetiginosa & Lavras-MG & 2005 \\
\hline
\end{tabular}

assepsia com hipoclorito de sódio (1\%) por 3 minutos e outra não.

O teste consistiu em dispor as sementes em placas do tipo gerbox, sobre duas folhas de papel do tipo germitest, previamente umedecidas com água destilada até saturação. Em seguida, as caixas foram acondicionadas em câmara de incubação, com temperatura de $20 \pm 2^{\circ} \mathrm{C}$ e fotoperíodo de $12 \mathrm{~h}$ de luz branca fluorescente/12h de escuro, por sete dias, quando então, efetuou-se a observação das estruturas fúngicas com auxílio de microscópio estereoscópico e ótico, identificando-se a população fúngica (16) pela comparação com as características descritas em literatura específica $(1,19)$.

Do mesmo modo que para o teste de sanidade, as amostras foram divididas em duas subamostras, sendo uma submetida à assepsia superficial com hipoclorito de sódio (1\%), durante três minutos, e a outra não. As sementes foram distribuídas sob papel tipo germitest em caixas plásticas tipo gerbox sendo o substrato previamente umedecido com água até a saturação. Em seguida foram colocadas em câmara de germinação tipo BOD, na temperatura de $30^{\circ} \mathrm{C}$, sob regime de luz constante, segundo Oliveira (24). Adicionou-se, a cada três dias, aproximadamente $5 \mathrm{~mL}$ de água/caixa durante a realização o experimento.

A avaliação foi realizada após 15 dias de instalação do teste. Obtevese a porcentagem de plântulas normais, anormais e sementes mortas. O critério adotado para avaliação baseou-se nas recomendações das Regras para Análise de Sementes (3), considerando-se germinadas as sementes que originaram plântulas normais, com todas as estruturas essenciais, com capacidade de produzirem plantas normais sob condições favoráveis de campo.

O delineamento estatístico utilizado foi o inteiramente casualizado, com 4 repetições de 50 sementes. Os dados obtidos no teste de germinação foram transformados utilizando arc sen. raiz quadrada de $\mathrm{x} / 100$, e submetidos à análise de variância. As médias dos tratamentos foram comparadas pelo teste de Tukey a $5 \%$ de probabilidade, usando o programa SANEST (29).

A transmissão de fungos associados às sementes de ipê-amarelo e ipê-roxo foi avaliada durante o teste de germinação, em plântulas normais e anormais, fazendo-se a contagem dos principais sintomas observados, nas amostras com e sem assepsia. Os dados não foram submetidos à análise de variância.

Foram retirados fragmentos lesionados de raiz, hipocótilo, epicótilo e folhas estes foram submetidos à assepsia com hipoclorito de sódio a $1 \%$, durante três minutos, e colocados sobre papel tipo germitest previamente umedecido com água destilada, em caixas plásticas tipo gerbox. Em seguida, foram mantidos em câmara de incubação, sob temperatura de $20 \pm 2{ }^{\circ} \mathrm{C}$ e luz alternada (12 horas de luz branca fluorescente/12 horas de escuro), durante sete dias, para avaliação.

Os fungos foram identificados através do exame individual feito com auxílio de microscópio estereoscópico e ótico, observando estruturas características dos fungos desenvolvidos sobre as lesões (1)

\section{RESULTADOS E DISCUSSÃO}

Foi verificado, neste experimento, que o teor de água das sementes encontrava-se entre 6 e 8,5\%, no momento da instalação dos testes. De acordo com Gemaque (9), as sementes do gênero Tabebuia são caracterizadas como ortodoxas para classificação fisiológica de armazenamento. Foi constatado que a conservação do teor de água em torno de $8 \%$ para as espécies ipê dourado, ipê-roxo e ipê-amarelo, em condições ideais de armazenamento, foi importante para manter a viabilidade dessas sementes por um ano e o teor crítico situou-se 
acima de $10 \%$ (12).

$\mathrm{Na}$ Tabela 2 encontram-se os fungos mais freqüentes, detectados nas diferentes amostras de ipê, e a taxa de redução após a assepsia. Observou-se que a assepsia reduziu, de forma significativa, a incidência da maioria dos fungos detectados, praticamente em todas as amostras avaliadas. Foi evidenciado que os fungos Aspergillus spp., Curvularia sp. e Trichothecium sp. Estavam sendo transportados pelos tecidos externos das sementes de ipê-amarelo e ipê-roxo, pois foram, praticamente erradicados das sementes após assepsia. Já os fungos Alternaria alternata, Cladosporium sp., Phoma sp., Phomopsis sp., Fusarium spp., Penicillium sp., Drechslera sp., Geotrichum sp. e Epicoccum sp., apesar de não terem sido erradicados das sementes, tiveram a incidência reduzida drasticamente após a assepsia. Esses resultados mostram que a maioria dos fungos estava contaminando e não infectando as sementes, pois considera-se contaminação a associação do patógeno a um tecido sem atividade enzimática, superficial ou interno, e uma infecção, quando ocorre em um tecido interno, com atividade vital (17).

Assume-se que a imersão em solução de hipoclorito de sódio a $1 \%$ por três minutos elimina os fungos contaminantes.

Esses resultados confirmam os obtidos por alguns autores que, realizando teste de sanidade em sementes florestais nativas, submetidas à assepsia, constataram que a maior parte dos fungos encontrados associados às sementes encontravam-se na superfície (2). Os fungos erradicados após a assepsia podem ter menor capacidade de sobrevivência em relação aos que são transportados internamente; estes podem sobreviver por mais tempo e ter maior probabilidade de causar doença na planta.

A eliminação completa de determinados fungos detectados pode depender de vários fatores, sendo alguns deles a localização na semente, a condição em que as sementes se encontram, concentração do produto e período de imersão no produto. Henning e França Neto (19) verificaram a erradicação de patógenos localizados internamente em sementes com elevados índices de danos mecânicos, provavelmente,

Tabela 2. Incidência de fungos em diferentes amostras de sementes de ipê-amarelo (Tabebuia serratifolia) e ipê-roxo (T. impetiginosa) com assepsia (C/ A) e sem assepsia (S/A) e taxa de redução (Tx) pela assepsia superficial.

(continua)

\begin{tabular}{|c|c|c|c|c|c|c|c|c|c|c|c|c|c|c|}
\hline & \multicolumn{14}{|c|}{ Fungos (\%) } \\
\hline & \multicolumn{3}{|c|}{ Cladosporium sp. } & \multicolumn{4}{|c|}{ Alternaria alternata } & \multicolumn{4}{|c|}{ Epicoccum sp. } & \multicolumn{3}{|c|}{ Phoma sp. } \\
\hline \multirow{2}{*}{$\begin{array}{l}\text { Amostras } \\
\text { AmrJ2 }\end{array}$} & S/A & $\mathrm{C} / \mathrm{A}$ & \multirow{2}{*}{$\begin{array}{l}\text { Tx } \\
90\end{array}$} & \multicolumn{2}{|c|}{ S/A } & $\mathrm{C} / \mathrm{A}$ & \multirow{2}{*}{$\begin{array}{l}\mathbf{T x} \\
96\end{array}$} & \multicolumn{2}{|c|}{ S/A } & $\mathrm{C} / \mathrm{A}$ & \multirow{2}{*}{$\begin{array}{l}x x \\
44\end{array}$} & \multirow{2}{*}{$\begin{array}{r}\text { S/A } \\
23 \text { bA }\end{array}$} & \multirow{2}{*}{$\begin{array}{l}\mathrm{C} / \mathrm{A} \\
3 \mathrm{abB}\end{array}$} & \multirow{2}{*}{$\begin{array}{l}\mathbf{T x} \\
87\end{array}$} \\
\hline & $87 \mathrm{aA}^{*}$ & $9 \mathrm{bB}$ & & 76 & $\mathrm{abA}$ & $3 \mathrm{bcB}$ & & 27 & $\mathrm{cA}$ & $15 \mathrm{abcA}$ & & & & \\
\hline AmrI2 & $88 \mathrm{aA}$ & $0 \mathrm{cB}$ & 100 & 83 & $\mathrm{abA}$ & $2 \mathrm{cB}$ & 98 & 40 & $\mathrm{bcA}$ & 8 bcB & 80 & $30 \mathrm{bA}$ & $0 \mathrm{bB}$ & 100 \\
\hline AmrI3 & $90 \mathrm{aA}$ & $7 \mathrm{bB}$ & 92 & 76 & $\mathrm{abA}$ & $12 \mathrm{abB}$ & 84 & 76 & $\mathrm{aA}$ & $23 \mathrm{aB}$ & 70 & $22 \mathrm{bA}$ & $7 \mathrm{abB}$ & 68 \\
\hline RoxL2 & $94 \mathrm{aA}$ & $8 \mathrm{bB}$ & 92 & 71 & $\mathrm{abA}$ & 4 abcB & 94 & 58 & $\mathrm{abA}$ & $23 \mathrm{aB}$ & 60 & 51 aA & $3 \mathrm{abB}$ & 94 \\
\hline RoxP3 & 86 aA & $10 \mathrm{abB}$ & 88 & 65 & bcA & 8 abcB & 88 & 22 & $\mathrm{cA}$ & 14 abcA & 36 & $53 \mathrm{aA}$ & $14 \mathrm{aB}$ & 74 \\
\hline RoxL3 & 89 aA & $6 \mathrm{bB}$ & 93 & 85 & $\mathrm{aA}^{*}$ & 8 abcB & 91 & 8 & $\mathrm{dA}$ & $4 \mathrm{cA}$ & 50 & $14 \mathrm{bA}$ & $9 \mathrm{abA}$ & 36 \\
\hline RoxG5 & $88 \mathrm{aA}$ & $4 \mathrm{bcB}$ & 96 & 49 & $\mathrm{cA}$ & 5 abcB & 90 & 27 & $\mathrm{cA}$ & 5 bcB & 81 & $15 \mathrm{bA}$ & $3 a b B$ & 80 \\
\hline RoxL5 & 88 aA & $24 \mathrm{aB}$ & 73 & 78 & $\mathrm{abA}$ & $15 \mathrm{aB}$ & 81 & 55 & bA & $16 \mathrm{abB}$ & 71 & $29 \mathrm{bA}$ & $7 \mathrm{abB}$ & 76 \\
\hline Média & $89 \mathrm{~A}$ & $8 \mathrm{~B}$ & 91 & 85 & $\mathrm{aA}$ & 8 abcB & 91 & 39 & A & $13 \mathrm{~B}$ & 67 & $30 \mathrm{~A}$ & $6 \mathrm{~B}$ & 80 \\
\hline $\mathrm{CV}$ & & & & & & & & & 17 & & & & & \\
\hline
\end{tabular}

*Médias seguidas pela mesma letra, minúscula, entre linhas e maiúsculas entre duas colunas contíguas, para cada fungo, não diferem entre si pelo teste de Tukey a $5 \%$

Tabela 2. Incidência de fungos em diferentes amostras de sementes de ipê-amarelo (Tabebuia serratifolia) e ipê-roxo (T. impetiginosa) com assepsia (C/ A) e sem assepsia (S/A) e taxa de redução (Tx) pela assepsia superficial.

(continuação)

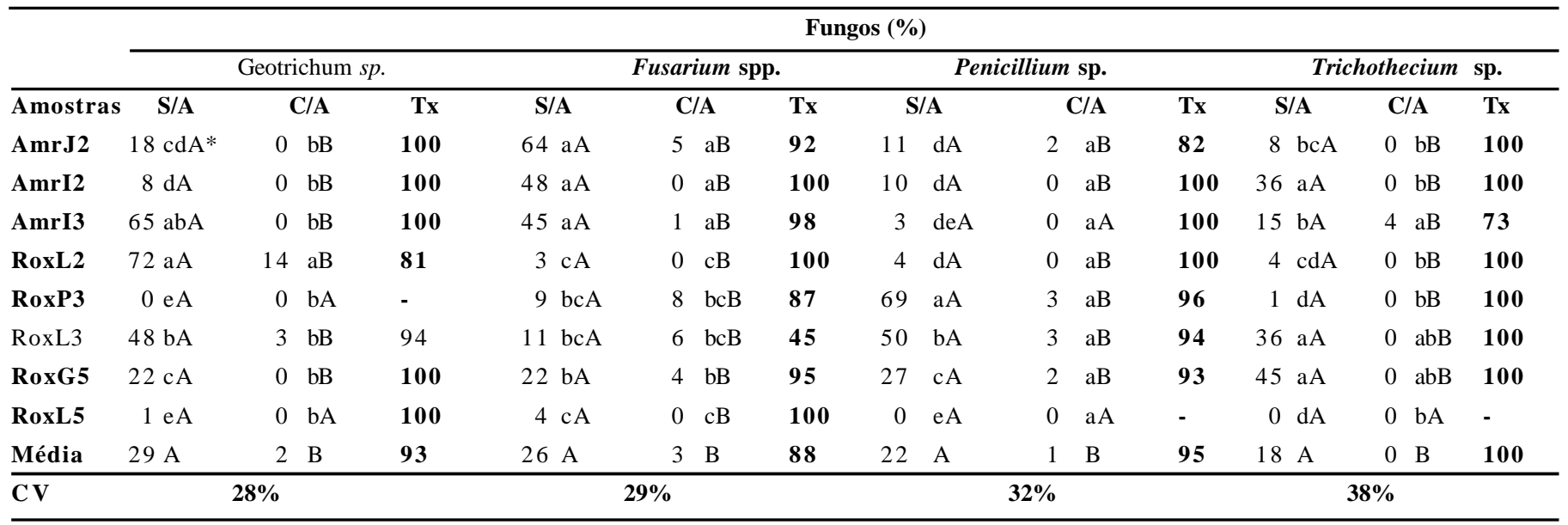

*Médias seguidas pela mesma letra, minúscula, entre linhas e maiúsculas entre duas colunas contíguas, para cada fungo, não diferem entre si pelo teste de Tukey a $5 \%$. 
Tabela 2. Incidência de fungos em diferentes amostras de sementes de ipê-amarelo e ipê-roxo com assepsia (C/A) e sem assepsia (S/A) e taxa de redução (Tx) pela assepsia superficial.

(continuação)

\begin{tabular}{|c|c|c|c|c|c|c|c|c|c|c|c|c|c|}
\hline \multirow{3}{*}{$\begin{array}{l}\text { / } \\
\text { Amostras }\end{array}$} & \multicolumn{13}{|c|}{ Fungos (\%) } \\
\hline & \multicolumn{4}{|c|}{ Phomopsis $s p$. } & \multicolumn{3}{|c|}{ Drechslera sp. } & \multicolumn{3}{|c|}{ Aspergillus spp. } & \multicolumn{3}{|c|}{ Curvularia sp. } \\
\hline & S/A & & /A & $\mathbf{T x}$ & S/A & $\mathrm{C} / \mathrm{A}$ & $\mathbf{T x}$ & S/A & $\mathrm{C} / \mathrm{A}$ & $\mathbf{T x}$ & S/A & $\mathrm{C} / \mathrm{A}$ & $\mathbf{T x}$ \\
\hline AmrJ2 & $7 \mathrm{cA}^{*}$ & 0 & $\mathrm{cB}$ & 100 & $11 \mathrm{bcdA}$ & $0 \mathrm{aB}$ & 100 & $1 \mathrm{cA}$ & $0 \mathrm{aB}$ & 100 & $1 \mathrm{bcdA}$ & $0 \mathrm{aA}$ & 100 \\
\hline AmrI2 & $0 \mathrm{dA}$ & 0 & $\mathrm{cA}$ & - & $21 \mathrm{abA}$ & $0 \mathrm{aB}$ & 100 & $0 \mathrm{cA}$ & 0 aA & - & $3 \mathrm{bcA}$ & $0 \mathrm{aB}$ & 100 \\
\hline AmrI3 & $8 \mathrm{cA}$ & 1 & $\mathrm{bcB}$ & 87 & $28 \mathrm{aA}$ & $2 \mathrm{aB}$ & 93 & $0 \mathrm{cA}$ & 0 aA & - & $7 \mathrm{bA}$ & $0 \mathrm{aB}$ & 100 \\
\hline RoxL2 & $0 \mathrm{dA}$ & 0 & $\mathrm{cA}$ & - & $5 \mathrm{dA}$ & $1 \mathrm{aA}$ & 80 & $0 \mathrm{cA}$ & 0 aA & - & $1 \mathrm{cdA}$ & $0 \mathrm{aA}$ & 100 \\
\hline RoxP3 & $0 \mathrm{dA}$ & 0 & $\mathrm{cA}$ & - & $6 \mathrm{dA}$ & $1 \mathrm{aB}$ & 83 & $0 \mathrm{cA}$ & $0 \mathrm{aA}$ & - & $30 \mathrm{aA}$ & $0 \mathrm{aB}$ & 100 \\
\hline RoxL3 & $15 \mathrm{cA}$ & 8 & bA & 27 & $0 \mathrm{dA}$ & $1 \mathrm{aB}$ & 94 & $9 \mathrm{bA}$ & $0 \mathrm{aB}$ & 100 & $0 \mathrm{dA}$ & $0 \mathrm{aA}$ & - \\
\hline RoxG5 & $41 \mathrm{bA}$ & 23 & $\mathrm{aB}$ & 37 & $5 \mathrm{dA}$ & $0 \mathrm{aB}$ & 100 & $58 \mathrm{aA}$ & $1 \mathrm{aB}$ & 98 & $4 \mathrm{bcA}$ & $0 \mathrm{aB}$ & 100 \\
\hline RoxL5 & 54 aA & 36 & $\mathrm{aB}$ & 33 & $8 \mathrm{cdA}$ & $0 \mathrm{aB}$ & 100 & $0 \mathrm{cA}$ & $0 \mathrm{aA}$ & - & $3 \mathrm{bcA}$ & $2 \mathrm{aA}$ & 33 \\
\hline Média & $16 \mathrm{~A}$ & 8 & B & 50 & $10 \mathrm{~A}$ & $1 \mathrm{~B}$ & 94 & $8 \mathrm{~A}$ & $0 \mathrm{~B}$ & 100 & $6 \mathrm{~A}$ & $0 \mathrm{~B}$ & 100 \\
\hline
\end{tabular}

* Médias seguidas pela mesma letra, minúscula entre linhas e maiúsculas entre duas colunas contíguas, para cada fungo, não diferem entre si pelo teste de Tukey a $5 \%$.

Tabela 3. Porcentagem de plântulas normais (PN), plântulas anormais (PA), sementes mortas (SM) e plântulas com sintomas (PCS) em amostras de ipêamarelo e ipê-roxo, com assepsia (C/A) e sem assepsia (S/A) submetidas ao teste de germinação.

\begin{tabular}{|c|c|c|c|c|c|c|c|c|}
\hline \multirow[t]{3}{*}{$\overline{\text { Amostras }}$} & \multicolumn{8}{|c|}{ Germinação e Sintomas em Plântulas (\%) } \\
\hline & \multicolumn{2}{|c|}{ PN } & \multicolumn{2}{|c|}{ PA } & \multicolumn{2}{|c|}{$\mathbf{S M}$} & \multicolumn{2}{|c|}{ PCS } \\
\hline & $\mathbf{S} / \mathbf{A}$ & C/A & S/A & C/A & S/A & C/A & S/A & C/A \\
\hline RoxL2 & 41 a $\mathrm{A}^{*}$ & $32 \mathrm{abA}$ & $40 \operatorname{abcdA}$ & $23 \mathrm{cB}$ & $19 \mathrm{cB}$ & 43 bc A & 14 & 9 \\
\hline RoxP3 & $35 \mathrm{aA}$ & 38 aA & $46 \mathrm{abA}$ & $47 \mathrm{abA}$ & $18 \mathrm{cA}$ & $15 \mathrm{dA}$ & 19 & 12 \\
\hline $\operatorname{Amr} \mathbf{J} 2$ & 34 a A & $16 \mathrm{bcdB}$ & $26 \mathrm{cdB}$ & $43 \mathrm{abA}$ & $39 \mathrm{ab} A$ & $40 \mathrm{bcA}$ & 13 & 0 \\
\hline AmrI3 & 32 a A & 23 abcA & $45 \mathrm{abcA}$ & $52 \mathrm{abA}$ & $22 \mathrm{bc} \mathrm{A}$ & $25 \mathrm{~cd} \mathrm{~A}$ & 9 & 8 \\
\hline RoxG5 & 32 a A & $9 \mathrm{cdB}$ & 26 bcdA & $23 \mathrm{cA}$ & $45 \mathrm{aB}$ & $68 \mathrm{aA}$ & 34 & 21 \\
\hline RoxL3 & 31 a $\mathrm{A}$ & $10 \mathrm{cdB}$ & $24 \mathrm{dA}$ & 32 bcA & $46 \mathrm{aA}$ & $56 \mathbf{a b A}$ & 20 & 12 \\
\hline AmrI2 & 31 a A & $34 \mathrm{aA}$ & 42 abcdA & $34 \mathrm{abcA}$ & $27 \mathrm{abcA}$ & $30 \mathrm{cdA}$ & 10 & 3 \\
\hline RoxL5 & 30 a A & $8 \mathrm{~d}$ B & $50 \mathrm{aA}$ & 55 aA & 19 c B & $35 \mathrm{cA}$ & 16 & 12 \\
\hline Média & 33 A & $21 \mathrm{~B}$ & $37 \mathrm{~A}$ & 39 A & $29 B$ & 39 A & 17 & 10 \\
\hline $\mathrm{CV} \%$ & & & & & & & & \\
\hline
\end{tabular}

* Medias seguidas de mesma letra, minúscula entre linhas e maiúsculas entre colunas, não diferem entre si pelo teste de Tukey a 5\%.

resultante da penetração do hipoclorito nas rachaduras existentes no tegumento.

Estudo realizado por Machado et al. (18), submetendo sementes de cedro (Cedrela fissilis ) e cerejeira (Eugenia involucrata) a diferentes concentrações e períodos de imersão em hipoclorito, constatou diferença no controle de fungos, sendo que hipoclorito a $10 \%$, por 5 minutos de exposição, apresentou o melhor desempenho. Zorato et al (28) relataram que, de acordo com o pH, concentração e período de submersão de sementes de soja por diferentes agentes químicos, ocorreu diferença relevante nos resultados, sendo que, dentre os produtos utilizados, o hipoclorito de sódio em diferentes concentrações, proporcionou redução de microrganismos infestantes e não a erradicação.

Pelos resultados obtidos, foi possível verificar que a gama de fungos incidentes nas sementes foi a mesma para as duas espécies e pouco relacionada com o local, período de coleta e armazenamento dessas sementes, embora a incidência dos fungos tenha variado para algumas amostras.

Os fungos detectados neste trabalho podem ser divididos em fungos de campo e de armazenamento. Os fungos do campo estabelecem-se na semente antes da colheita, ou seja, no período do seu crescimento e maturação. Após as sementes serem colhidas e armazenadas, estão sujeitas a invasão por um grupo de fungos designados como de armazenamento (30).

Em relação aos fungos de armazenamentos, Aspergillus spp. e Penicillium sp., são relatados por diversos autores como os principais gêneros de fungos associados às sementes durante o armazenamento em condições inadequadas $(8,21)$. Aspergillus spp. foi detectado, em maior incidência, na amostra RoxG5 e, para as demais, a incidência foi muito baixa ou nula. Verificou-se a presença de Penicillium sp. em todas as amostras, exceto em RoxL5. As maiores incidências foram constatadas nas amostras RoxP3 e RoxL3, havendo diferença estatística entre elas. Nas demais amostras a incidência foi baixa, variando de $4 \%$ a $27 \%$.

Dentre os fungos identificados, considerados de campo, encontramse Cladosporium sp. Alternaria alternata, Epicoccum sp., Phoma sp., Geotrichum sp., Fusarium sp., Penicillium sp., Trichothecium sp., Phomopsis sp., Drechslera sp., Aspergillus spp. e Curvularia sp. 
Os resultados do teste de germinação de sementes de ipê-amarelo e ipê-roxo estão na Tabela 3.

A assepsia causou redução, em média, de $64 \%$ na germinação das sementes. Esta redução foi observada nas amostras AmrJ2, RoxG5, RoxL3 e RoxL5; as demais amostras não diferiram em relação à assepsia. Isto demonstra que o procedimento provocou fitotoxicidade àquelas amostras, dificultando a avaliação dos efeitos dos fungos sobre a germinação das sementes.

Sementes de ipê apresentam taxa de germinação em torno de $60 \%$ (11). Neste trabalho, os resultados obtidos evidenciam que as sementes de ipê apresentaram baixa germinação, sendo de 30 a $41 \%$. Esses valores baixos de germinação podem ter sido influenciados pela grande quantidade de fungos detectados nas sementes (conforme Tabela 1), que podem ter ocasionado anormalidades (média de 37\%) nas plântulas originadas dessas sementes. Degan et al. (7) verificaram que sementes de ipê-branco, recém-colhidas no estádio maduro, apresentaram germinação máxima de 56,7\%. Alguns autores, como Lorenzi (14), relataram que sementes de ipê germinam em baixa porcentagem.

Lopes et al. (15) e Castellani et al. (4) observaram que a contaminação das sementes pode afetar, de forma severa, a qualidade fisiológica e, em alguns casos, inibir por completo a capacidade germinativa das sementes.

Entretanto, Oliveira et al (23) comparando métodos de desinfestação de sementes de canafístula (Peltophorum dubium), detectaram, principalmente, Trichoderma sp., Penicillium sp., Aspergillus niger e Fusarium sp., e verificaram que a porcentagem de sementes infeccionadas não comprometeu a germinação. Isto confirma que nem sempre a associação de fungos com sementes acarreta doença ou queda na qualidade fisiológica. Porém, esta associação pode favorecer a sobrevivência do fungo e sua disseminação.

Além da incidência de fungos nas sementes, outros fatores podem afetar a germinação. No presente trabalho a baixa umidade em que as sementes de ipê encontravam-se $(6,0 \%$ a $8,5 \%)$ pode ter facilitado a rápida absorção, do produto utilizado para assepsia, ocorrendo dano de embebição durante o processo.

Além do dano por embebição, a concentração do produto utilizada e o tempo de imersão, provavelmente, tenham prejudicado a germinação, causando anormalidades e aumentando a porcentagem de sementes mortas. Trabalho realizado por Sales (26), avaliando o efeito de produtos químicos no controle de fungos em sementes de ipêamarelo e ipê-roxo, constatou que o hipoclorito de sódio a $1 \%$ por 10 minutos, utilizado na assepsia, provocou redução no tamanho, população, peso da matéria verde e da matéria seca de plântulas, mostrando ser prejudicial. Redução significativa na porcentagem de germinação de ipê-amarelo, causada pelo hipoclorito a $2 \%$ por três minutos, também foi, observada, por Nery (22). Oliveira (24) porém, não observou efeitos negativos quando sementes de ipê-amarelo e ipêroxo foram submetidas à assepsia. com hipoclorito de sódio a $2 \%$ durante 2 minutos.

Para a avaliação da transmissão dos fungos por sementes de ipê, em todas as amostras avaliadas, além da contagem de plântulas normais, anormais e sementes mortas, também foi quantificado o número de plântulas que apresentaram sintomas. Embora não tenha-se coletado os dados para análise estatística, a maior porcentagem de sintomas foi observada nas amostras sem assepsia (Tabela 3 ).

Os principais sintomas observados foram necrose e/ou manchas escuras nos primeiros pares de folhas, hipocótilo, epicótilo e raízes. Nas folhas, os fungos observados foram Alternaria alternata, Phoma sp. e Phomopsis sp.; no hipocótilo e epicótilo foram encontrados, associados às lesões, Alternaria alternata, Phoma sp., Phomopsis sp., Fusarium spp., Aspergillus spp. e Penicillium sp.; e na raiz Phoma sp., Fusarium spp., Penicillium sp., Trichothecium sp. e Alternaria alternata.

Não foi verificada diferença nos tipos de sintomas nas amostras com e sem assepsia e não ocorreu uma associação constante de um determinado tipo de sintoma com um fungo específico.

\section{REFERÊNCIAS BIBLIOGRÁFICAS}

1. Barnett, H.L.; Hunter, B.B. Illustrated genera of imperfect fungi, 4th ed. Saint Paul: The American Phytopathological Society, $1998.218 \mathrm{p}$.

2. Botelho, L. S.; Grandis, A.; Nascimento, W. M. O.; Moraes, M. H. D. de; Menten, J. O. .M.. Incidência de fungos associados às sementes de espécies florestais nativas da Amazônia. . In: Simpósio Brasileiro de Patologia de Sementes, 8, 2004, João Pessoa. Palestras e Resumos... João Pessoa, 2004, p.196.

3. Brasil. Ministério da Agricultura e Reforma Agrária. Regras para análise de sementes. Brasília: CLAV; DNDV; SNAD; MA, 1992. 365p.

4. Castellani, E. D.; Silva, A.; Barreto, M.; Aguiar, I. B., Influência do tratamento químico na população de fungos e na germinação de sementes de Bauhinia variegata $L$. var variegata. Revista Brasileira de Sementes, Brasília, v.18, n.1, p. 4144, 1996.

5. Carneiro, J.S. Micoflora associada às sementes de essências florestais em Paraopeba, MG. Fitopatologia Brasileira, Brasília, v. 11, p.556-557, 1986.

6. Carvalho, W.L. de; Muchovej, J.J. Fungos associados a sementes de essências florestais. Revista Árvore, Viçosa, v.15, n.2, p.173$178,1991$.

7. Degan, P.; Aguiar, I. B.; Sader, R.; Perecin, D.; Pinto, L. R. Influência de métodos de secagem na conservação de sementes de ipê-branco. Revista Brasileira de Engenharia Agrícola e Ambiental, Campina Grande, v.5, n.3, p.492-496, 2001.

8. Dhingra, O.D. Prejuízos causados por microorganismos durante o armazenamento de sementes. Revista Brasileira de Sementes, Brasília, v.7, n.1, p.139-145, 1985.

9. Gemaque, R. C. R. Maturação, tolerância à dessecação e alterações na qualidade fisiológica em sementes de ipê-roxo (Tabebuia impetiginosa (Mart.)Satandl. envelhecidas artificialmente. 1999 p.93. Dissertação (Mestrado em Ciências Florestais) Universidade Federal de Lavras, Lavras, 1999.

10. Henning, A.A.; França Neto, J.B. Problemas na avaliação de germinação de sementes de soja com alta incidência de Phomopsis sp. Revista Brasileira de Sementes, Brasília, v.2, n. 3, p. 922, mar. 1980 .

11.Instituto de Pesquisa e Estudos Florestais.IPEF: Disponível em http://www.ipef.br/identificação/tabebuia.asp: Acesso em:10 ago. 2006.

12.Kageyama, P. Y.; Marquez, F. C. M. Comprotamento de sementes de curta longevidade armazenadas com diferentes teores de umidade inicial: gênero Tabebuia. IN: Reunion Sore Problemas Em Semillas Florestales Tropicales, 1980, San Felipe-Bacalar. Memória...México: INIF, 1981.v.1,p.3747-352. Publicación Especial,35.

13.Lasca, C.C.; Sampaio, A P.; Cintra, A.F. Condições fitossanitárias de sementes importadas de Pinus spp. O Biológico, São Paulo, v.37, n.11, p.287-292, nov. 1971 .

14.Lorenzi, H. Árvores brasileiras: manual de identificação e cultivo de plantas arbóreas nativas do Brasil. Nova Odessa: Editora Plantarum, 1998. 368p.

15.Lopes, J.C.; Jardim, I.C.C; Sobreira, D.G.; Forde, G.H.A.; Tatagiba, J.S. Associação entre germinação, vigor e sanidade em sementes de milho precoce e normal, produzidos na área experimental do Centro Agropecuário da UFES. In: Congresso Brasileiro De Sementes, 7, Campo Grande, 1991. Informativo ABRATES, Brasília, v.1, n.4, p.55, 1991. (Resumos). 
16.Lucca Filho, O. Metodologia dos testes de sanidade de sementes. In: SoAve, J.; WetZel, M.M.V.S., (Ed). Patologia de Sementes Campinas, Fundação Cargill, 1987. p.430-440.

17. Machado, J. da C. Tratamento de sementes: fundamentos aplicações. Brasília: MEC-ESAL-FAEPE, 1988. 106p.

18.Machado, A.A.; Muniz, M.F.B. Hoppe, J.M.; Camargo, R. Influência de diferentes tratamentos se sementes de cedro (Cedrela fissilis Vell.) e cerejeira (Eugenia involucrata DC.) sobre a incidência de fungos de armazenamento. Fitopatologia Brasileira, Brasília, v.29, p354,.2004(suplemento)

19.Marthur, S. B.; Kongsdal, O. Common laboratory seed health testing methods for detectine fungi. Basserdorf: International Seed Testing Assoication, 2003. 425p.

20.Mucci, E.S.F.; Lasca, C.C. Flora fúngica de sementes de essências florestais nativas. Fitopatologia Brasileira, v.11, n.2, p.352-353, 1986.

21.Neergaard, P. Seed pathology. London: MacMillan Press, v.1, 839p. 1979

22.Nery, M. Aspectos morfofisiológicos do desenvolvimento de sementes de Tabebuia serratifolia Vahl Nich. 2005. 95p.Dissertação (Mestrado em Fitotecnia) Universidade Federal de Lavras, Lavras, 2005.

23. Oliveira, L.M,; Davide, A .C.; Carvalho, M.L.M. Avaliação de métodos para quebra da dormência e para desinfestação de sementes de canafístula (Peltophorum dubium (Sprengel) Taubert. Revista Árvore, Viçosa, v.27, n.5, p.597-603, 2003.

24.Oliveira, L. M. Avaliação da qualidade de sementes de Tabebuia serratifolia Vahl Nich. e T. impetiginosa (Martius Ex A.p. de
Candolle Standley) envelhecidas natural e artificialmente. 2004. p. 160 Tese (Doutorado em Fitotecnia) Universidade Federal de Lavras, Lavras, 2004.

25.Parisi, J. J. D.; Martins, M. C.; Sales, W. R. M. Qualidade sanitária e fisiológica de sementes de espécies florestais do estado de São Paulo. Campinas, SP,2003 In: SIMPÓSIO Brasileiro De Patologia De Sementes, 8, 2004, João Pessoa. Palestras e Resumos... João Pessoa, 2004. p.203.

26.Sales, N. L. Efeito da população fúngica e do tratamento químico no desempenho de sementes de ipê-amarelo, ipê-roxo e barbatimão. 1992. 89p. Dissertação (Mestrado em Fitopatologia). Universidade Federal de Lavras, Escola Superior de Agricultura de Lavras, Lavras, 1992.

27.Souza, V.C.; Bruno, R. L.A.; Araújo, E.; Andrade, L.A. Sanidade de sementes armazenadas de Tabebuia serratifolia (Vahl.) Nich. . In: Simpósio Brasileiro de Patologia de Sementes, 8, 2004: João Pessoa. Palestras e Resumos... João Pessoa, 2004. p.235.

28.Zorato, M. F.; Homechin, M.; Henning, A. A. Efeitos da assepsia superficial com diferentes agentes químicos na incidência de microrganismos em sementes de soja. Revista Brasileira de Sementes, Campinas, v.23, n.1, p.159-166, 2001.

29.Zonta, E. F.; Machado, A. A.; Silveira Júnior, P. Sistema de análise estatística (SANEST) para microcomputador (versão 1.0). In: Simpósio de Estatística Aplicada à Experimentação Agronômica, 1985, Piracicaba, Anais...Piracicaba: ESALQ, p. 74-90, 1985.

30.Wetzel, M.M.V. da S. Fungos do armazenamento. In: Soave, J. E Wetzel, M.M.V.S. (Ed.). Patologia de sementes. Campinas: Fundação Cargill, 1987. cap. 9, p.260-274. 